\title{
Far-IR spectra of small neutral gold clusters in the gas phase
}

Philipp Gruene ${ }^{\mathrm{a}}$, Burkhard Butschke ${ }^{\mathrm{a}, \mathrm{b}}$, Jonathan T. Lyon ${ }^{\mathrm{a}, \mathrm{c}}$, David M. Rayner, ${ }^{\mathrm{d}}$ and André Fielicke $\mathrm{e}^{\mathrm{a}, \mathrm{e}^{*}}$

a) Fritz-Haber-Institut der Max-Planck-Gesellschaft, Faradayweg 4-6, D-14195 Berlin, Germany

b) present address: Department of Organic Chemistry, Weizmann Institute of Science, 76100 Rehovot, Israel

c) Department of Natural Sciences, Clayton State University, 2000 Clayton State Blvd., Morrow, Georgia 30260, USA

d) National Research Council, 100 Sussex Drive, Ottawa, Ontario, Canada K1A OR6

e) Institut für Optik und Atomare Physik, Technische Universität Berlin, Hardenbergstr. 36, D-10623 Berlin, Germany,

\begin{abstract}
Vibrational spectra of small neutral gold clusters containing up to $8 \mathrm{Au}$ atoms are measured in the far-infrared $\left(46-222 \mathrm{~cm}^{-1}\right)$ via photodissociation of their complexes with krypton atoms. Comparisons with calculated IR spectra for bare $\mathrm{Au}_{n}$ clusters using density functional theory allow for structural assignment. For these small sizes, all clusters are found to be planar and of comparably high symmetry. For Aur no data is available, as this cluster size is not detected in the photoionization mass spectra due to its high ionization energy. The structures assigned are for $n=4$ : rhombus $\left(\mathrm{D}_{2 \mathrm{~h}}\right)$; 5: trapezoid $\left(\mathrm{C}_{2 \mathrm{v}}\right)$; 7: edge-capped triangle $\left(\mathrm{C}_{\mathrm{s}}\right)$, 8: 4-fold edge-capped square $\left(\mathrm{D}_{4 \mathrm{~h}}\right)$.
\end{abstract}

\footnotetext{
*Email: fielicke@physik.tu-berlin.de
} 


\section{Introduction}

Detailed knowledge of the geometric and electronic structures of clusters is a prerequisite for understanding the evolution of their properties with size. This is particularly the case for systems where novel non-bulk like properties emerge at the nano-scale. Examples for such observations are the transition to insulator or even van-der-Waals bound clusters for certain metals [1,2], the optical properties of metal clusters or nano-particles embedded in glass $[3,4]$, or the appearance of unprecedented chemical reactivity below a particular particle size. The discovery of high catalytic activity of gold nano-particles for low temperature $\mathrm{CO}$ oxidation [5] likewise has triggered many investigations of the physical and chemical properties of gas-phase gold clusters including their structures [69].

Most experimental investigations sensitive to structural details of gold clusters have been performed on charged species due to the possibility of easy mass separation and thereby size-selection. These studies give insight into an interesting structural evolution that does not have a direct parallel in other metals: the transition from 2- to 3-dimensional structures at comparably large sizes [7,8]. For the cationic clusters, the smallest cluster with a 3D geometry is Aus, while for the anions one finds the crossover at the 12-atom cluster with co-existing 2D and 3D structures. For neutral clusters one might intuitively expect the transition to occur somewhere in between. The only experimentally confirmed structures are those of $\mathrm{Au}_{4}, \mathrm{Au}_{7}$ (2D) and $\mathrm{Au}_{19}, \mathrm{Au}_{20}$ (3D) [10,11], leaving a large unexplored gap. There have been many theoretical studies addressing this topic. For the neutrals, the small sizes are found to be planar [12-19]. In contrast to what can be inferred from the studies on the ions, there have been some predictions that the dimensionality change for the neutrals may occur at or even below $\mathrm{Au}_{8}$ [20-22]. It is evident, however, that the transition size as well as the predicted ground state structures, highly depend on the theoretical methods and how they treat relativistic effects and dispersion interactions. Several studies have explicitly focussed on Au8, and in particular more recent $\operatorname{CCSD}(\mathrm{T})$ calculations using comparably large basis sets find a $2 \mathrm{D}$ configuration as ground state for Au8, while the energy differences to the lowest 3D structure are only small [2328].

There are only few direct experimental studies on neutral gold clusters in the gas phase. Their ionization potentials have been determined by threshold electron impact ionization [29], excited states of $\mathrm{Au}_{3}$ have been characterized via resonant two-photon ionization spectroscopy [30], and for the same size, a vibronic spectrum in the mid-IR has been measured in a Ne matrix [31]. Optical spectra of odd-sized clusters $\operatorname{Au}_{n}(n=7,9,11,13)$ have been measured using photodissociation of weakly bound complexes with Xe atoms [32] as well as for mass-selectively deposited small $(n=1$ 5, 7-9) Au clusters in Ne matrices [33]. The latter results have been compared to results of TD-DFT calculations that reproduce several experimental features, but do not allow with certainty the attribution of the measured spectra to specific isomers. Vibrational data for neutral gold clusters as formed via electron detachment from the anions have been obtained via vibrationally resolved photoelectron spectroscopy [34-37]. It is, however, not clear that these are the ground state structures of the neutrals, taken the charge state dependence of the gold-cluster structures into account. By using slow-electron velocity-map imaging (SEVI) [38], transitions to single vibrational levels of $\mathrm{Au}_{2}$ and $\mathrm{Au}_{4}$ have been recently observed $[39,40]$. We have used photodissociation of rare-gas complexes to obtain the vibrational spectra in the far-IR $[10,11]$. In combination with quantum chemical calculations, such vibrational spectra can give detailed structural information for metal clusters.

In the following we report experimental far-IR spectra for neutral gold clusters up to Au8. While the spectra for $n=3,4$, and 7 have been discussed before [10,11], we now include Au5 and Aus. No experimental data is available for $\mathrm{Au}_{6}$ because it is not detected due to its high ionization energy. We compare IR multiple photon dissociation (IR-MPD) spectra of the $\mathrm{Au}_{n} \mathrm{Kr}_{m}$ complexes with calculated harmonic IR spectra obtained using density functional theory (DFT) for the bare clusters 
Aun. It will be shown that for the species discussed here, this is sufficient for the determination of the gold cluster structures. A detailed discussion of the effects of rare-gas ligands and anharmonicity (i.e. through finite temperature effects and fluctionality) has been given elsewhere for $n=3,4,7$ [11].

\section{Methods}

\section{Experimental}

The experiments are performed in a molecular beam machine (see Fig. 1) connected to a beamline of the Free Electron Laser for Infrared eXperiments (FELIX) [41]. The set-up is a different one than used in most of our earlier experiments on metal clusters[42,43], implementing a continuous gas expansion laser ablation source. This is essential to form a stable distribution of $\mathrm{Au}_{n}-\mathrm{Kr}$ complexes. The set-up follows the design of Campargue [44] with a moderate vacuum ( 0.1 mbar) in the source chamber obtained by a mechanical booster / rotary vane pump combination (Edwards EH1200/E2M80), differential pumping stage $\left(10^{-4} \mathrm{mbar}\right)$, and a high vacuum stage $\left(10^{-6} \mathrm{mbar}\right)$ that contains a reflectron time-of-flight mass spectrometer (Jordan TOF Products, Inc.); the latter two are pumped by turbomolecular pumps.

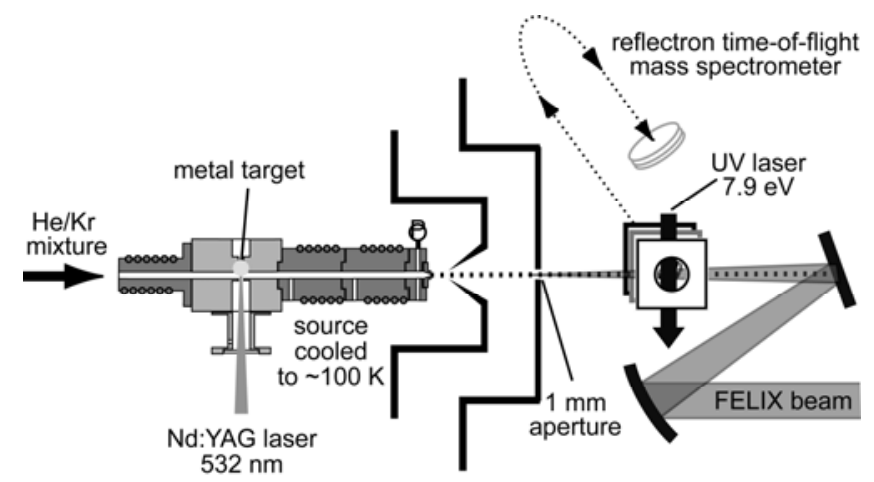

Fig. 1. Experimental set-up for far-IR multiple photon dissociation spectroscopy of neutral gold cluster-krypton complexes.

Gold clusters are produced by pulsed $(10 \mathrm{~Hz})$ laser ablation of a solid gold target in a continuous stream of a carrier gas, consisting of $1.5 \%$ krypton in helium. The total gas flow was about 1600 sccm, leading to a stagnation pressure of $\sim 10 \mathrm{mbar}$ in the source channel. The complete cluster source [45] is cooled to $\sim 100 \mathrm{~K}$ by liquid nitrogen flowing continuously through the tubing soldered onto the copper extensions of the central source part where ablation occurs. The clusters are efficiently thermalized in the source and weakly bound complexes with $\mathrm{Kr}$ atoms are formed. The cluster beam emitted by the source is skimmed and passes through a $1 \mathrm{~mm}$ diameter circular aperture. Neutral clusters are photoionized by $7.9 \mathrm{eV}$ photons from an $\mathrm{F}_{2}$ laser. The aperture defines the overlap between the cluster beam and the counter-propagating IR laser beam from FELIX. The ionization laser is timed to ionize the cluster package that has been irradiated with the $\sim 10 \mu$ s long IR pulse while flying through the weak focus close to the aperture. This corresponds to a delay between IR and UV pulses of approximately $50 \mu \mathrm{s}$. 
For a given IR wavenumber typically 600-1000 single mass spectra are averaged and the same number of reference mass spectra, with the IR laser off, are taken on alternating shots. The IR laser is tuned between 46 and $222 \mathrm{~cm}^{-1}$, typical pulse energies are on the order of $20-40 \mathrm{~mJ}$, and the bandwidth of the IR radiation is about $1-2 \%$ of the central wavelength. Size-specific far-IR spectra of the neutral cluster species are obtained by monitoring the IR induced changes in the intensity of the cations $I^{+}$. The relative intensity changes of the ionized species $I^{+}(v) / I^{+} 0$ are evaluated as a function of wavenumber $v$ As the ionization yield of a particular cluster species $I^{+} / I^{\text {neut }}$ is constant as long as saturation effects are avoided this equals the relative change in the neutral species. Relative absorption cross sections are calculated using: $v(v)=-\ln \left[I^{+}(v) / I^{+}\right] / F(v)$.

The normalization with the IR photon fluence $F(v)$ accounts for its variations over the tuning range. More technical details on far-IR multiple photon dissociation of metal cluster-rare gas complexes have been given before $[42,43]$.

\section{Theoretical}

For comparison with the experimental spectra, IR spectra are calculated for the various low-energy isomers of the pure $\mathrm{Au}_{n}$ clusters that have been suggested in the literature [12-28]. Computations are performed within TURBOMOLE V6.4 [46] using density functional theory employing the def2TZVP basis set (with the associated effective core potential def2-ecp) [47] and the Tao-PerdewStaroverov-Scuseria meta-GGA functional [48]. The suitability of such type of functionals for the investigation of gold clusters has been discussed before $[49,50]$.

The calculated harmonic vibrational frequencies are uniformly scaled by a constant multiplication factor of 1.06 that is chosen based on the comparison of experimental and theoretical peak positions for the gold clusters. Scaling of the calculated (harmonic) vibrational frequencies is performed to compensate for the anharmonicity of the vibrations, the neglect of electron correlation, or the use of incomplete basis sets [51]. Therefore, the optimal vibrational scaling factors depend on details of the theoretical methods [52]. Scaling factors larger than 1 we encounter also in studies of other systems, like pure and doped Si clusters $[53,54]$ and we attribute their need to a systematic underestimation of the calculated bond strength, due to shortcomings in our DFT approach.

\section{Results and discussion}

\section{Ionization efficiencies of $\mathrm{Au}_{n} \mathrm{Kr}_{m}$.}

Figure 2 shows mass spectra obtained by single photon ionization of the neutral cluster distribution at $7.9 \mathrm{eV}$. The observed intensity pattern for $\mathrm{Au}_{n}{ }^{+}$follows a strong odd/even oscillation with intense signals for the odd sized clusters and no or very little signals for $n=4,6$, 8, while $\mathrm{Kr}$ complexes are observed for all sizes except $n=6$. From $n=9$ on, all bare gold clusters and the Kr complexes are efficiently ionized. For the bare clusters, this follows largely the known experimental vertical ionization energies that had been determined via threshold electron impact ionization [29].

$\mathrm{The} \mathrm{Kr}$ complexes are expected to have a lower ionization energy (IE) compared to the bare clusters by approximately the strength of the charge-induced dipole interaction in the cationic $\mathrm{Au}_{n} \mathrm{Kr}^{+}$com- 
plex [55]. The precise interaction energy will depend on the structure of the charged metal cluster, but can be approximated to be on the order of $0.2 \mathrm{eV}$ per $\mathrm{Kr}$ atom. This brings the IE of $\mathrm{Au}_{4} \mathrm{Kr}_{2}$ and $\mathrm{Au}_{8} \mathrm{Kr}_{2} / 3$ into the range of the photon energy of the ionization laser. The IE of $\mathrm{Au}_{6}, 8.8 \mathrm{eV}$, is the highest for all clusters in the investigated size range and neither the ionization of the bare clusters nor its $\mathrm{Kr}$ complexes are observed. The small intensities observed for bare $\mathrm{Au}_{4}^{+}$and $\mathrm{Au}_{8}{ }^{+}$might be due to a hot fraction of neutral clusters. They are not due to fragmentation of the ionized Kr complexes, as their intensity does not depend on the IR wavelength.

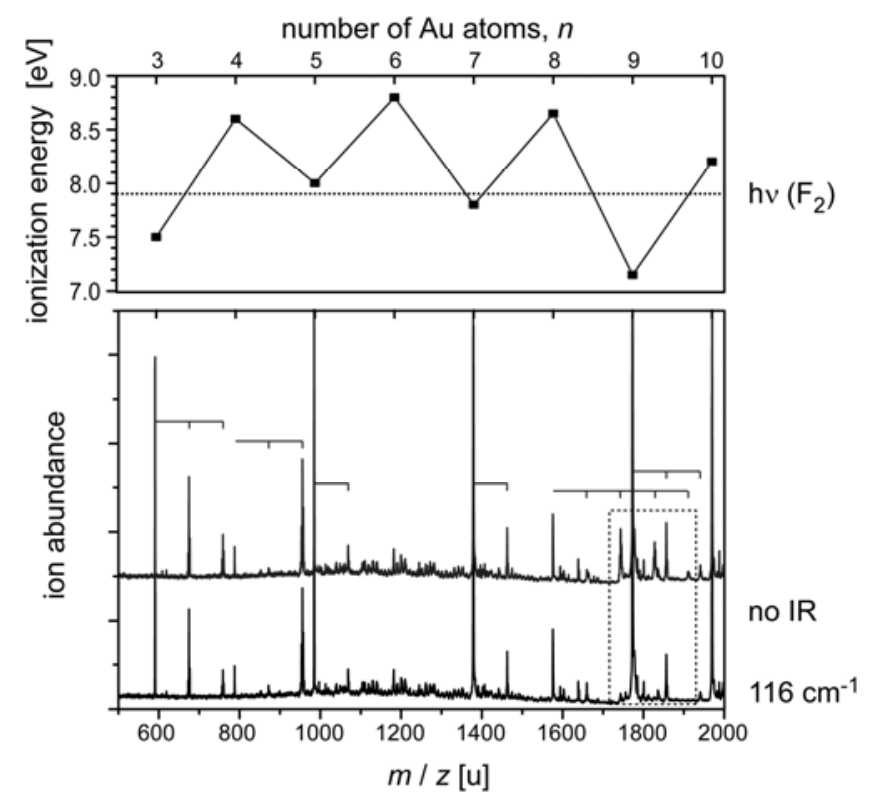

Fig. 2. Mass spectra of $\mathrm{Au}_{n} \mathrm{Kr}$ complexes ionized with $7.9 \mathrm{eV}$ photons (bottom) compared to the ionization energies of bare $\mathrm{Au}_{n}$ clusters (top). The bars mark the sequences of bare clusters and the corresponding $\mathrm{Kr}$ complexes. The upper mass spectrum is taken without IR irradiation, while for the lower one the IR is tuned to a resonance in the $\mathrm{Au}_{8} \mathrm{Kr}_{n}$ complexes that are depleted (see the marked mass range). Ionization of residual pump oil leads to some background signal in the 1000-1500 u range.

\section{Far-IR spectra}

Multiple photon dissociation spectra are recorded in the $46-222 \mathrm{~cm}^{-1}$ range. The lower limit was given by the performance of FELIX, while at higher frequencies no fundamental vibrations of the gold clusters are expected. This is in agreement with the predicted vibrational frequencies, see below. Note, for the neutral dimer $\omega_{\mathrm{e}}$ is $190.9 \mathrm{~cm}^{-1}$ [52] and only very few bands are observed above this value for the higher clusters (Fig. 3). The spectra of the different cluster sizes are rather dissimilar, demonstrating that they are fingerprints of the clusters' individual structures. The very similar appearance of the spectra of $\mathrm{Au}_{8} \mathrm{Kr}_{2}$ and $\mathrm{Au}_{8} \mathrm{Kr}_{3}$ illustrates that the addition of $\mathrm{Kr}$ atoms has no significant effect on the spectra. The case of $\mathrm{Au}_{3} \mathrm{Kr} / \mathrm{Au}_{3} \mathrm{Kr}_{2}$, where the spectra differ considerably, is somehow special, as the $\mathrm{Kr}$ atoms are comparatively strongly bound to $\mathrm{Au}_{3}$ and lead to a change from a slightly obtuse (in $\mathrm{Au}_{3} \mathrm{Kr}$ ) to an acute (in $\mathrm{Au}_{3} \mathrm{Kr}_{2}$ ) geometry; furthermore the presence and position of the bands is affected by anharmonic effects. This observation has been discussed in detail elsewhere [11], and the experimental spectra are only included here for completeness. 


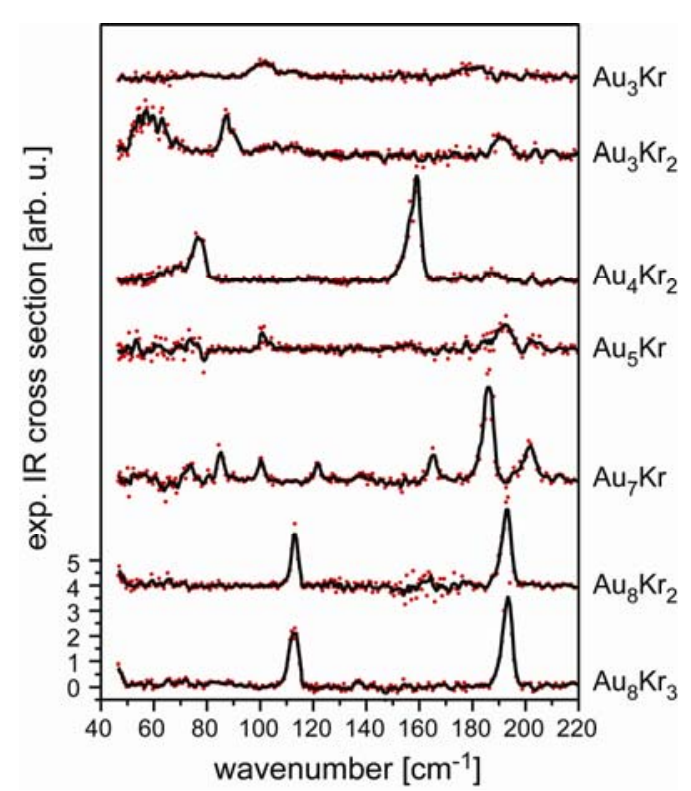

Fig. 3. IR-MPD spectra of krypton complexes of $\mathrm{Au}_{3}-\mathrm{Au} 8$. The dots are the measured data points, the lines are their 5-point running average. The spectra are all plotted on the same intensity scale and offset for clarity.

\begin{abstract}
$\mathrm{Au}_{4}$
For the tetramer, only the complex with two $\mathrm{Kr}$ atoms is observed in the mass spectrum. Its IR spectrum fits the predictions for the rhombic isomer 4a well, both in terms of band position and relative intensities (Fig. 4). The Y-shaped isomer $\mathbf{4 b}$ is found to be isoenergetic at the level of theory used here, but experimentally, there are no signs of any contribution to the spectrum. However, it has been predicted that $\mathbf{4 b}$ has a higher ionization potential than $\mathbf{4 a}$ [11,18], and its complexes (if present) may not get ionized by the $\mathrm{F}_{2}$ laser. The observed bands at $76 \mathrm{~cm}^{-1}$ (calc.: $78.5 \mathrm{~cm}^{-1}$ ) and 158 $\mathrm{cm}^{-1}\left(159.1 \mathrm{~cm}^{-1}\right)$, attributed to the 4a isomer, are in-plane vibrations of the central Au-dimer against the outer two atoms.

The here not observed Y-isomer can be formed by photodetachement from anionic $\mathrm{Au}_{4}^{-}$that is known to have this shape [56]. Using vibrationally resolved photoelectron spectroscopy the frequencies of three vibrational modes at $17(7), 97(7)$, and $171(7) \mathrm{cm}^{-1}$ of the resulting neutral have been determined and assigned to this Y-isomer [40]. We also observe good agreement with the values obtained for isomer $\mathbf{4 b}$ at the TPSS/def2-TZVP level of theory $\left(14.4 \mathrm{~cm}^{-1} \mathrm{~b}_{2} ; 91.6 \mathrm{~cm}^{-1} \mathrm{a}\right.$; $\left.164.8 \mathrm{~cm}^{-1} \mathrm{a}_{1}\right)$.
\end{abstract}




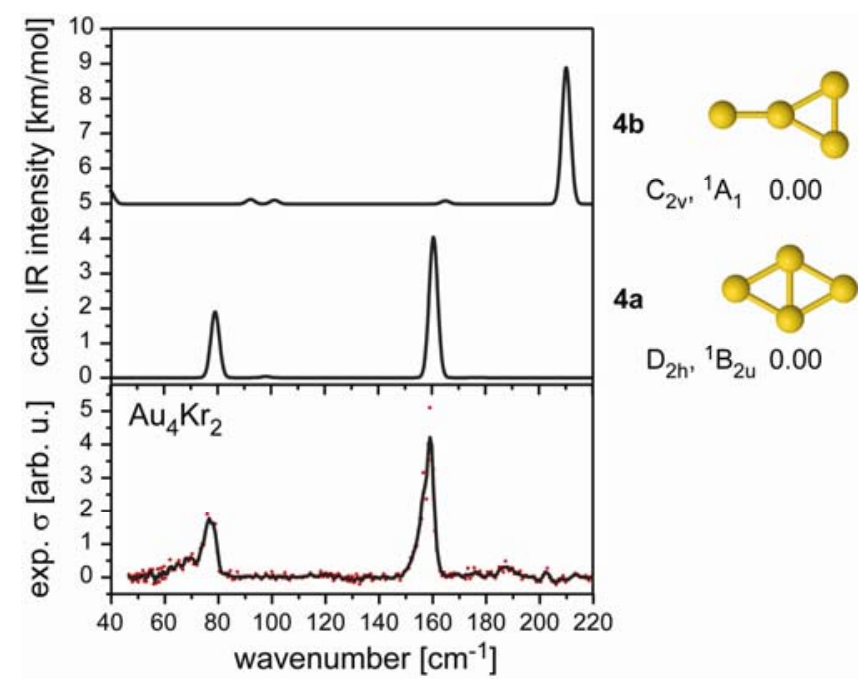

Fig. 4. Comparison of the IR-MPD spectrum of $\mathrm{Au}_{4} \mathrm{Kr}_{2}$ with calculated spectra for low energy isomers of Au4. Relative energies of the isomers are given in $\mathrm{eV}$ and include zero point vibrational energy corrections. The calculated line spectra are folded with a Gaussian line-width function of $3 \mathrm{~cm}^{-1}$ full width at half maximum for better visual comparison with the experimental spectra.

\section{$\mathrm{Au}_{5}$}

The far-IR-MPD spectrum of $\mathrm{Au}_{5} \mathrm{Kr}$ has an unfavourable signal-to-noise ratio (cf. the comparison with the other IR-MPD spectra in Fig. 1) and the bands are not very pronounced, as shown in Figure 3. This may be related to the low intensity of the species in the mass spectrum. Comparison with calculated spectra (Fig. 5) shows that the only definite band in the experimental spectrum at 192 $\mathrm{cm}^{-1}$ coincides with the most intense and highest frequency mode at $192.1 \mathrm{~cm}^{-1}$ of the calculated ground state isomer 5a, a planar trapezoidal structure (Fig. 5). The presence of the X-shaped isomer can be excluded as it does not fit the experimental spectrum and it is significantly higher in energy.

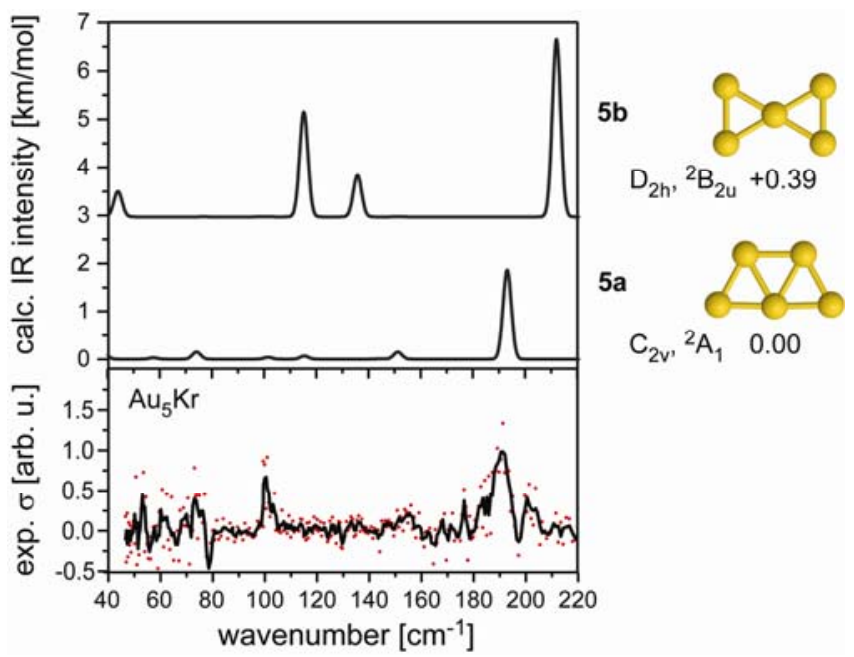

Fig. 5. Comparison of the IR-MPD spectrum of $\mathrm{Au}_{5} \mathrm{Kr}$ with calculated spectra for low energy isomers of Aus. Relative energies of the isomers are given in $\mathrm{eV}$ and include zero point vibrational energy corrections. 
For $\mathrm{Au}_{6}$, no experimental data is available. The mass spectrum contains no signals for this cluster size, neither for the bare cluster, nor for its krypton complexes. This can be attributed to the very high ionization potential of this cluster, which is measured to be $8.8 \mathrm{eV}$ [29] and thus well above the photon energy used here for ionization $(7.8 \mathrm{eV})$. The predicted ground state structure is a planar triangle $\left(\mathrm{D}_{3 \mathrm{~h},}{ }^{1} \mathrm{E}^{\prime}\right)$.

\section{Aur}

The IR-MPD spectrum of the 7-atom cluster has been discussed before, and it has been shown that its structure is a planar triangle with an additional atom bound to one of its edges [10,11]. The harmonic spectrum shown in Fig. 6 is in good agreement with the experimental multiple photon dissociation spectrum of $\mathrm{Au} 7 \mathrm{Kr}$. However, some deviations in the band intensities and a slight broadening of the experimentally determined highest frequency bands can be noted. It has been suggested that these effects are related to the rare gas binding [10,57], but recent calculations explicitly considering dispersion interactions, find that they are due to anharmonic effects caused by the fluctional behaviour at the experimental temperature [11]. Modelling of the finite temperature IR spectrum via molecular dynamics simulations can well explain the experimental features. At elevated temperatures, the dynamics involves self-isomerization via the $C_{2 v}$ symmetric structure of the anion into a geometry equivalent to the starting structure.

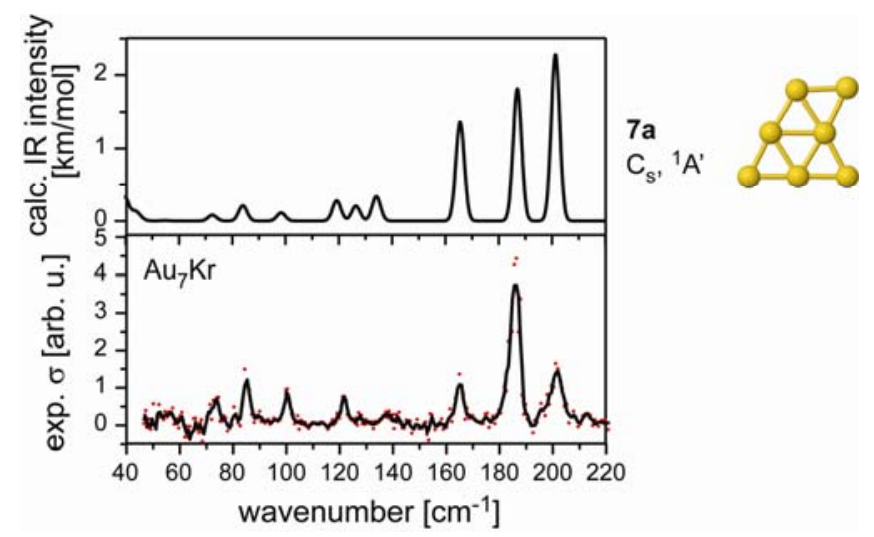

Fig. 6. Comparison of the IR-MPD spectrum of $\mathrm{Au} 7 \mathrm{Kr}$ with the calculated spectrum of the ground state isomer of Au7.

$\mathrm{Au}_{8}$

In the following, only the experimental spectrum of $\mathrm{Au}_{8} \mathrm{Kr}_{3}$ is discussed, as it is very similar to the spectrum of $\mathrm{Au}_{8} \mathrm{Kr}_{2}$ but has a slightly better quality. It shows the onset of a band at the lowfrequency end of the spectrum $\left(\sim 46 \mathrm{~cm}^{-1}\right)$ as well as two rather pronounced lines at $113 \mathrm{~cm}^{-1}$ and $193 \mathrm{~cm}^{-1}$. As seen in Fig. 7, this agrees well with the calculated band positions $(42.2,110.7,194.6$ $\mathrm{cm}^{-1}$ ) for the planar star-shaped $\mathrm{D}_{4 \mathrm{~h}}$ ground state geometry 8a, although the relative intensity of the second band is significantly too low in the computed spectrum. This might be an anharmonic effect or due to the influence of the rare gas atoms. In the Kr complexes, the symmetry is reduced, which may lead to splitting of degenerate modes (not observed here) or the appearance of bands that are initially not IR-allowed. The latter may be the case for the small feature in the experimental spec- 
trum at $137 \mathrm{~cm}^{-1}$ that fits well to an IR-inactive mode at $136.2 \mathrm{~cm}^{-1}$ in the $\mathbf{8 a}$ structure. A second planar isomer $\mathbf{8 b}$ and all 3D-structures $\mathbf{8 c}-\mathbf{f}$ are significantly higher in energy and do not match the experimental IR spectrum. While inclusion of dispersion forces in the calculations is expected to lower the relative energies of the $3 \mathrm{D}$ isomers, the assignment to the planar star-shaped $\mathrm{D}_{4 \mathrm{~h}}$ structure is unambiguous.

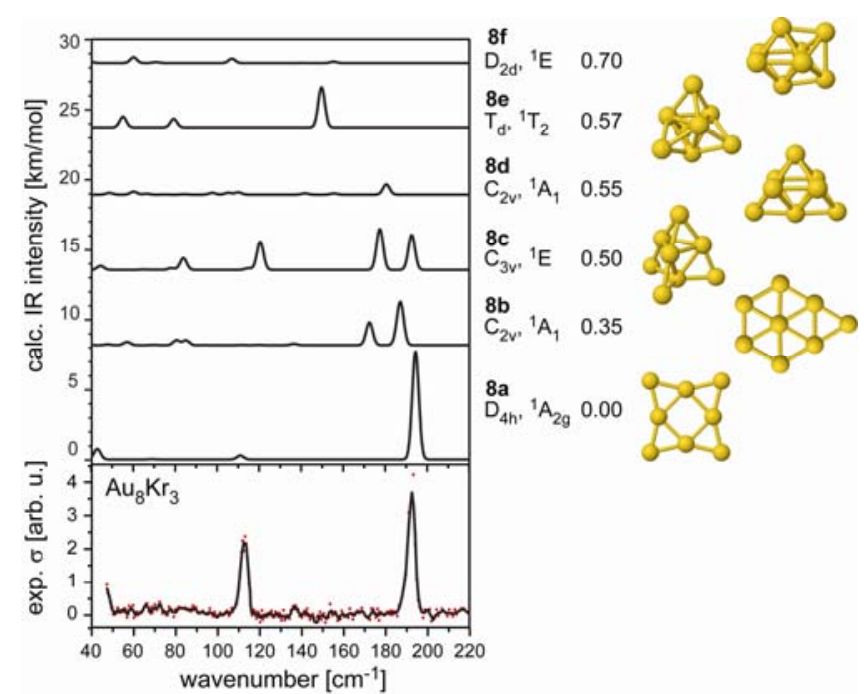

Fig. 7. Comparison of the IR-MPD spectrum of $\mathrm{Au}_{8} \mathrm{Kr}_{3}$ with calculated IR spectra of low energy isomers of Au8. All calculated spectra are plotted on the same intensity scale, but offset for clarity. Relative energies of the isomers are given in eV and include zero-point vibrational energy corrections.

\section{Conclusions}

The far-IR spectra of small neutral gold clusters complexed with krypton atoms $\mathrm{Au}_{n} \mathrm{Kr}_{m}(n=3-5,7$, 8 ) are presented. For $n=5,7,8$ the comparison with calculated harmonic spectra of the bare $\mathrm{Au}_{n}$ clusters results in good agreement for the lowest energy isomers at the TPSS/def2-TZVP level of theory. In the case of $\mathrm{Au}_{4}$, the assigned structure is one of two calculated isoenergetic lowest-energy isomers, while the second one is not observed, possibly due to its higher ionization energy. The second isomer is found to be formed via electron detachment from anionic Au4 ${ }^{-}$[40]. All of the identified structures are fully planar, showing that, contrary to some theoretical predictions, neutral gold clusters remain 2-dimensional until at least Aus. While rare gas binding and anharmonicity effects can have an influence on the observed far-IR spectra of $\mathrm{Au}_{n} \mathrm{Kr}_{m}$ [11,57], for the sizes discussed here, unambiguous assignment is possible by considering the harmonic vibrational spectra of the bare Aun.

\section{Supplementary information}

Cartesian coordinates of the gold cluster structures and (unscaled) vibrational frequencies of all isomers.

Acknowledgements. We gratefully acknowledge the "Stichting voor Fundamental Onderzoek der Materie" (FOM) in providing beam time on FELIX and the skilful assistance of the FELIX staff, in particular A.F.G. van der Meer and B. Redlich. This work is supported by the Cluster of Excellence "Unifying Concepts in Catalysis" coordinated by the Technical University Berlin and funded by the Deutsche Forschungsgemeinschaft (DFG). J.T.L. acknowledges support from the Alexander-von-Humboldt-Stiftung. We thank G. Meijer for his continued support. 


\section{References}

1. Rademann, K.: Photoionization mass spectrometry and valence photoelectron-photoion coincidence spectroscopy of isolated clusters in a molecular beam. Ber. Bunsenges. Phys. Chem. 931989653

2. Issendorff, B. V., Cheshnovski, O.: Metal to insulator transitions in clusters. Ann. Rev. Phys. Chem. 562005549

3. Eichelbaum, M., Rademann, K., Müller, R., Radtke, M., Riesemeier, H., Görner, W.: On the chemistry of gold in silicate glasses: studies on a nonthermally activated growth of gold nanoparticles. Angew. Chem. Int. Ed. 4420057905

4. Eichelbaum, M., Rademann, K., Hoell, A., Tatchev, D. M., Weigel, W., Stößer, R., Pacchioni, G.: Photoluminescence of atomic gold and silver particles in soda-lime silicate glasses. Nanotechnology 192008135701

5. Haruta, M., Kobayashi, T., Sano, H., Yamada, N.: Novel Gold catalysts for the oxidation of carbon monoxide at a temperature far below $0{ }^{\circ} \mathrm{C}$. Chem. Lett. 161987405

6. Bernhardt, T. M.: Gas-phase kinetics and catalytic reactions of small silver and gold clusters. Int. J. Mass Spectrom. 24320051

7. Schooss, D., Weis, P., Hampe, O., Kappes, M. M.: Determining the size-dependent structure of ligand-free gold-cluster ions. Phil. Trans. R. Soc. A 36820101211

8. Wang, L.-M., Wang, L.-S.: Probing the electronic properties and structural evolution of anionic gold clusters in the gas phase. Nanoscale 420124038

9. Woodham, A. P., Fielicke, A., in D.M.P. Mingos (Ed.), Structure \& Bonding: Gold Clusters, Colloids and Nano-Particles, in press

10. Gruene, P., Rayner, D. M., Redlich, B., Van Der Meer, A. F. G., Lyon, J. T., Meijer, G., Fielicke, A.: Structures of neutral Au7, $\mathrm{Au}_{19}$, and $\mathrm{Au}_{20}$ clusters in the gas phase. Science 3212008674

11. Ghiringhelli, L. M., Gruene, P., Lyon, J. T., Rayner, D. M., Meijer, G., Fielicke, A., Scheffler, M.: Not so loosely bound rare gas atoms: finite-temperature vibrational fingerprints of neutral gold-cluster complexes. New J. Phys. 152013083003

12. Bravo-Pérez, G., Garzón, I. L., Novaro, O.: Ab initio study of small gold clusters. J. Mol. Struct.: THEOCHEM 4931999225

13. Grönbeck, H., Andreoni, W.: Gold and platinum microclusters and their anions: comparison of structural and electronic properties. Chem. Phys. 26220001

14. Bonacic-Koutecký, V., Burda, J., Mitric, R., Ge, M., Zampella, G., Fantucci, P.: Density functional study of structural and electronic properties of bimetallic silver-gold clusters: Comparison with pure gold and silver clusters. J. Chem. Phys. 11720023120

15. Fernández, E. M., Soler, J. M., Garzón, I. L., Balbás, L. C.: Trends in the structure and bonding of noble metal clusters. Phys. Rev. B 702004165403

16. Remacle, F., Kryachko, E. S.: Structure and energetics of two- and three-dimensional neutral, cationic, and anionic gold clusters $\mathrm{Au}_{5 \leq n \leq 9^{\mathrm{z}}}(\mathrm{z}=0, \pm 1)$. J. Chem. Phys. 1222005044304

17. Assadollahzadeh, B., Schwerdtfeger, P.: A systematic search for minimum structures of small gold clusters $\mathrm{Au}_{n}\left({ }_{n}=2--20\right)$ and their electronic properties. J. Chem. Phys. 1312009064306

18. Lee, H. M., Kim, K. S.: Observable structures of small neutral and anionic gold clusters. Chem. Eur. J. 18201213203

19. Götz, D. A., Schäfer, R., Schwerdtfeger, P.: The performance of density functional and wavefunction-based methods for $2 \mathrm{D}$ and 3D structures of $\mathrm{Au}_{10}$. J. Comp. Chem. 3420131975

20. Häkkinen, H., Landman, U.: Gold clusters $\mathrm{Au}_{N},(2 \leq N \leq 10)$ and their anions. Phys. Rev. B 622000 R2287

21. Wang, J., Wang, G., Zhao, J.: Density-functional study of $\mathrm{Au}_{n}(n=2-20)$ clusters: Lowest-energy structures and electronic properties. Phys. Rev. B 662002035418

22. Olson, R. M., Varganov, S., Gordon, M. S., Metiu, H., Chretien, S., Piecuch, P., Kowalski, K., Kucharski, S. A., Musial, M.: Where does the planar-to-nonplanar turnover occur in small gold clusters? J. Am. Chem. Soc. 12720051049

23. Grönbeck, H., Broqvist, P.: Comparison of the bonding in Aus and Cu8: A density functional theory study. Phys. Rev. B 712005 073408

24. Han, Y.-K.: Structure of Aus: Planar or nonplanar? J. Chem. Phys. 1242006024316

25. Diefenbach, M., Kim, K. S.: Spatial structure of Aus: Importance of basis set completeness and geometry relaxation. J. Phys. Chem. B 110200621639

26. Olson, R. M., Gordon, M. S.: Isomers of Aus. J. Chem. Phys. 1262007214310

27. Serapian, S. A., Bearpark, M. J., Bresme, F.: The shape of Aus: gold leaf or gold nugget? Nanoscale 520136445

28. Hansen, J. A., Piecuch, P., Levine, B. G.: Communication: Determining the lowest-energy isomer of Aus: 2D, or not 2D. J. Chem. Phys. 1392013

29. Jackschath, C., Rabin, I., Schulze, W.: Electron impact ionization potentials of gold and silver clusters $\mathrm{Me}_{n}, n \leq 22$. Ber. Bunsenges. Phys. Chem. 9619921200

30. Bishea, G. A., Morse, M. D.: Resonant two-photon ionization spectroscopy of jet-cooled Au3. J. Chem. Phys. 9519918779

31. Guo, R., Balasubramanian, K., Wang, X., Andrews, L.: Infrared vibronic absorption spectrum and spin-orbit calculations of the upper spin-orbit component of the Aus ground state. J. Chem. Phys. 11720021614

32. Collings, B. A., Athanassenas, K., Lacombe, D., Rayner, D. M., Hackett, P. A.: Optical absorption spectra of Au7, Au9, Au11, and $\mathrm{Au}_{13}$, and their cations: Gold clusters with 6, 7, 8, 9, 10, 11, 12, and 13 s-electrons. J. Chem. Phys. 10119943506

33. Lecoultre, S., Rydlo, A., Felix, C., Buttet, J., Gilb, S., Harbich, W.: UV-visible absorption of small gold clusters in neon: Aun $(n$ $=1-5$ and 7-9). J. Chem. Phys. 1342011074302

34. Ho, J., Ervin, K. M., Lineberger, W. C.: Photoelectron spectroscopy of metal cluster anions: $\mathrm{Cu}_{n}{ }^{-}, \mathrm{Ag}_{n}{ }^{-}$, and $\mathrm{Au}_{n}^{-}$. J. Chem. Phys. 9319906987

35. Gantefor, G. F., Cox, D. M., Kaldor, A.: High resolution photodetachment spectroscopy of jet-cooled metal cluster anions: Au2and $\mathrm{Ag}_{3}{ }^{-}$. J. Chem. Phys. 9319908395

36. Gantefor, G. F., Cox, D. M., Kaldor, A.: Zero electron kinetic energy spectroscopy of Au6- J. Chem. Phys. 9619924102

37. Handschuh, H., Ganteför, G., Eberhardt, W.: Vibrational spectroscopy of clusters using a "magnetic bottle" electron spectrometer. Rev. Sci. Instr. 6619953838 
38. Neumark, D. M.: Slow Electron Velocity-map imaging of negative ions: Applications to spectroscopy and dynamics. J. Phys. Chem. A 112200813287

39. León, I., Yang, Z., Wang, L.-S.: High resolution photoelectron imaging of Au2². J. Chem. Phys. 1382013184304

40. Yang, Z., León, I., Wang, L.-S.: Communication: Vibrational spectroscopy of Au4 from high resolution photoelectron imaging. J. Chem. Phys. 1392013021106

41. Oepts, D., Van Der Meer, A. F. G., Van Amersfoort, P. W.: The Free-Electron Laser User Facility FELIX. Infrared Phys. Technol. 361995297

42. Fielicke, A., Kirilyuk, A., Ratsch, C., Behler, J., Scheffler, M., Von Helden, G., Meijer, G.: Structure determination of isolated metal clusters via far-infrared spectroscopy. Phys. Rev. Lett. 932004023401

43. Fielicke, A., Von Helden, G., Meijer, G.: Far-Infrared spectroscopy of isolated transition metal clusters. Eur. Phys. J. D 342005 83

44. Campargue, R.: High Intensity Supersonic Molecular Beam Apparatus. Rev. Sci. Instr. 351964111

45. Fielicke, A., Rademann, K.: Interaction of Bismuth Oxide Cluster Cations with Alkenes and Molecular Oxygen: $\mathrm{Bi}_{4} \mathrm{O}_{6}{ }^{+}$, a Possible Reactive Center for Alkene Oxidation. J. Phys. Chem. A 10420006979

46. Ahlrichs, R., Bär, M., Häser, M., Horn, H., Kölmel, C.: Electronic structure calculations on workstation computers: The program system TURBOMOLE. Chem. Phys. Lett. 1621989165

47. Weigend, F., Ahlrichs, R.: Balanced basis sets of split valence, triple zeta valence and quadruple zeta valence quality for $\mathrm{H}$ to Rn: Design an assessment of accuracy. Phys. Chem. Chem. Phys. 720053297

48. Tao, J., Perdew, J. P., Staroverov, V. N., Scuseria, G. E.: Climbing the density functional ladder: Nonempirical meta-generalized gradient approximation designed for molecules and solids. Phys. Rev. Lett. 912003146401

49. Johansson, M. P., Lechtken, A., Schooss, D., Kappes, M. M., Furche, F.: 2D-3D transition of gold cluster anions resolved. Phys. Rev. A 772008053202

50. Mantina, M., Valero, R., Truhlar, D. G.: Validation study of the ability of density functionals to predict the planar-to-threedimensional structural transition in anionic gold clusters. J. Chem. Phys. 1312009064706

51. Meier, R. J.: Calculating the vibrational spectra of molecules: An introduction for experimentalists with contemporary examples. Vib. Spectrosc. 43200726

52. Merrick, J. P., Moran, D., Radom, L.: An evaluation of harmonic vibrational frequency scale factors. J. Phys. Chem. A 1112007 11683

53. Lyon, J. T., Gruene, P., Fielicke, A., Meijer, G., Janssens, E., Claes, P., Lievens, P.: Structures of silicon cluster cations in the gas phase. J. Am. Chem. Soc. 13120091115

54. Ngan, V. T., Gruene, P., Claes, P., Janssens, E., Fielicke, A., Nguyen, M. T., Lievens, P.: Disparate effects of Cu and V on structures of exohedral transition metal-doped silicon clusters: A combined far-infrared spectroscopic and computational study. J. Am. Chem. Soc. 132201015589

55. Knickelbein, M. B., Menezes, W. J. C.: Metal cluster-rare gas van der Waals complexes: $\mathrm{Nb}_{n} \mathrm{Ar}_{m}$ and $\mathrm{Fe}_{n} \mathrm{Kr}_{m}$. Chem. Phys. Lett. 1841991433

56. Furche, F., Ahlrichs, R., Weis, P., Jacob, C., Gilb, S., Bierweiler, T., Kappes, M. M.: The structures of small gold cluster anions as determined by a combination of ion mobility measurements and density functional calculations. J. Chem. Phys. 1172002 6982

57. Mancera, L. A., Benoit, D. M.: Towards an understanding of the vibrational spectrum of the neutral Au7 cluster. Phys. Chem. Chem. Phys. 1520131929 